\title{
Electron mobility in (100) homoepitaxial layers of phosphorus-doped diamond
}

\author{
I. Stenger, ${ }^{\text {a) }}$ M.-A. Pinault-Thaury, N. Temahuki, R. Gillet, S. Temgoua, H. Bensalah, E. Chikoidze, Y. Dumont, \\ and J. Barjon \\ Université Paris-Saclay, UVSQ, CNRS, GEMaC, 78000 Versailles, France
}

(Dated: 17 February 2021)

The electron transport in $n$-type diamond is investigated using a series of (100) homoepitaxial layers doped with phosphorus in the range $10^{16}-10^{18} \mathrm{~cm}^{-3}$. The electrical properties of the $n$-type layers, such as electron concentration and mobility, were measured using the resistivity and Hall effect as a function of temperature. The scattering of electrons in the diamond was modeled for the (100) orientation, which is preferred for electronic device applications. The physical parameters extracted from the fitting of the experimental data allow us to discuss the upper limit for the electron mobility in (100) $n$-type diamond.

PACS numbers: XXXXXXXXX

\section{INTRODUCTION}

Among wide bandgap semiconductors, diamond has attracted a significant amount of research interest because of its exceptional physical properties, such as its high breakdown voltage, its high thermal conductivity, and its high carrier mobilities. ${ }^{1}$ These properties make diamond a very promising material for high-temperature, high-power, and high-frequency electronic applications. Like semiconductors $\mathrm{ZnO}$ and $\mathrm{GaN}$, the control of its doping is asymmetric. The $p$-type doping of diamond by substituting boron for carbon is fairly easy and now technologically mature. ${ }^{2-4}$ In contrast, the $n$-type conductivity of diamond is difficult to realize and remains an issue for the fabrication of diamond-based bipolar devices.

The conventional crystalline orientations used for $n$ type doping are (111) and (100). Due to the ready formation of crystalline defects on (111) growth surfaces, better electronic properties are generally observed on (100) homoepilayers. For instance, at room temperature, the Hall mobility of holes in boron-doped diamond is $532 \mathrm{~cm}^{2} \mathrm{~V}^{-1} \mathrm{~s}^{-1}$ for the (111) orientation but reaches $2010 \mathrm{~cm}^{2} \mathrm{~V}^{-1} \mathrm{~s}^{-1}$ for the (100) orientation. ${ }^{5,6}$ As to the compensation ratio $\eta=N_{D} / N_{A}$ for p-type diamond, where $N_{D}$ and $N_{A}$ are the donor and acceptor concentrations, it remains low for both orientations $(\eta=3 \%$ at best for (111) and $\eta<10^{-6}$ for $\left.(100)^{6,34}\right)$. Furthermore, (100) diamond substrates are more easily polished and less expensive to produce. Thus, at the moment, doping of orientation (100) is used to integrate $n$-type diamond in bipolar devices.

With an ionization energy of $0.6 \mathrm{eV}$, phosphorus donors give the highest $n$-type conductivities reported so far. ${ }^{7}$ Though, in (100) phosphorus-doped diamond, the conductivities $^{8}$ are limited by high compensation ratios, $k=N_{A} / N_{D}$ for n-type diamond. In (100) homoepilayers,

a) Author to whom correspondence should be addressed: ingrid.stenger@uvsq.fr indeed, the compensation is usually higher $(k \geq 40 \%)^{9,10}$ than in (111) homoepilayers $(k<15 \%) .{ }^{11}$ This results in a large amount of ionized impurities, which are known to have a detrimental impact on the mobility of electrons. Another limitation of the (100) orientation is that phosphorus atoms are not always fully incorporated when substituting for carbon atoms. ${ }^{10}$ Thus, the scattering that limits the free electron mobility in (100) phosphorusdoped diamond needs to be understood in more detail.

In this work, we report on the Hall mobility of (100) phosphorus-doped diamond films with a wide range of phosphorus concentrations, from $3.5 \times 10^{16}$ to $7.2 \times$ $10^{18} \mathrm{~cm}^{-3}$. The temperature dependence of the electron Hall mobility is compared with theoretical calculations modeled for the (100) epilayers.

\section{EXPERIMENTS}

Four phosphorus-doped diamond thin films were homoepitaxially grown on the polished (100) surfaces of synthetic type Ib diamond crystals of size $3 \times 3 \times 0.5 \mathrm{~mm}^{3}$, with a miscut angle of $3.5^{\circ}$, as measured by $\mathrm{x}$-ray diffraction. The films were grown by microwave plasmaassisted chemical vapor deposition using a liquid organic compound, tertiarybutylphosphine, as the phosphorus precursor. ${ }^{12}$ The main deposition conditions are described elsewhere, ${ }^{13}$ except for the $(\mathrm{P} / \mathrm{C})$ gas ratio, which varied from 0.12 to $12 \%$, and the growth temperature, which was in the range $1000-1150^{\circ} \mathrm{C}$. Samples A, B, and $\mathrm{D}$ were grown over $1.5 \mathrm{~h}$ and sample $\mathrm{C}$ over $3 \mathrm{~h}$. Secondary ion mass spectrometry (SIMS; Cameca IMS 7f) with $\mathrm{Cs}^{+}$primary ions accelerated to $10 \mathrm{keV}$ was used to measure the depth distribution of $\mathrm{P}, \mathrm{B}, \mathrm{N}$, and $\mathrm{H}$ atoms in the diamond films. The concentrations of $\mathrm{P}$ and $\mathrm{B}$ were quantified using implanted standards.

DC electrical measurements were made with our own high-temperature and high-impedance setup. Van der Pauw resistivity and Hall effect measurements were employed to determine the resistivity, carrier type, density, and mobility in the temperature range $300-850 \mathrm{~K}$ and 
(a)

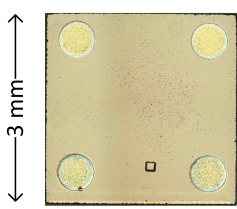

(b)

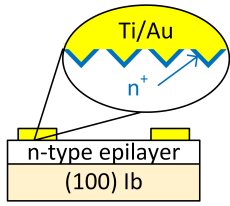

(c)

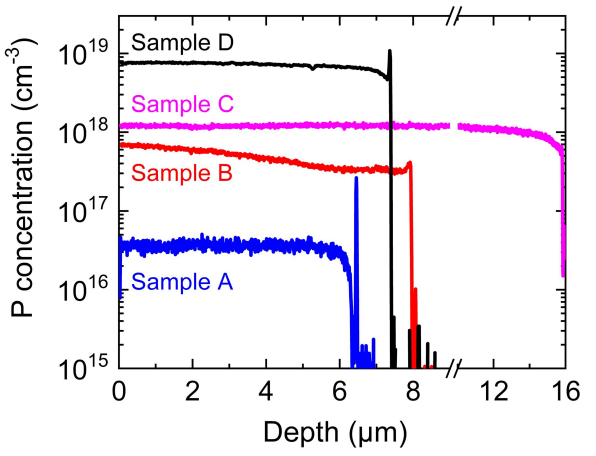

FIG. 1. (a) Optical image of sample D. (b) Schematic cross section of the electrical contacts. (c) SIMS depth profiles of the phosphorus concentration in homoepitaxial diamond layers A, B, C, and D.

under a DC magnetic field of $1.6 \mathrm{~T}$. The Hall scattering factor was assumed to be 1. Quasi-Ohmic contacts were obtained by selectively growing highly phosphorus-doped diamond on the (111) surface of Ni-etched microstructures, followed by $\mathrm{Ti} / \mathrm{Au}$ film deposition on top, as shown in our previous work. ${ }^{14}$ Figures $1(\mathrm{a})$ and $1(\mathrm{~b})$ show an optical image of a typical sample and a schematic cross section of the electrodes, respectively.

Cathodoluminescence spectroscopy was used to evaluate the optical activity of the phosphorus dopants in the epilayers. This technique can quantify the phosphorus donor concentration $N_{D}$, regardless of the concentration of compensating acceptors $N_{A}$. It is based on the intensity ratio between the bound and free exciton luminescence signals at low temperatures $(10 \mathrm{~K})$, following the method detailed in Refs. 15 and 16, which describe the experimental setup.

\section{RESULTS AND DISCUSSION}

Figure 1(c) shows the SIMS depth profiles of the phosphorus concentration of samples A, B, C, and D. The phosphorus concentration $[\mathrm{P}]$ of our epilayers is quite homogeneous in depth and has a sharp interface layer/substrate. Regarding the usual unintentional impurities in chemical vapor deposition, the hydrogen concentration is at the detection limit of SIMS (around $2 \times 10^{18} \mathrm{~cm}^{-3}$ ) while the boron concentration is below the detection limit of SIMS (around $1 \times 10^{15} \mathrm{~cm}^{-3}$ ). The thickness of the epilayers ranges from 6.5 to $15.8 \mu \mathrm{m}$. $N_{D}$ values obtained by cathodoluminescence spectroscopy for samples A, B, and $\mathrm{C}$ are, respectively, $3.5 \times 10^{16}$,
TABLE I. Sample characteristics: epilayer thickness $(d)$, phosphorus donor concentration $\left(N_{D}\right)$, compensation ratio $(k)$, the corresponding concentration of compensating acceptors $\left(N_{A}\right)$, and activation energy $\left(E_{A}\right)$.

\begin{tabular}{cccccc}
\hline \hline Sample & $d(\mu \mathrm{m})$ & $N_{D}\left(\mathrm{~cm}^{-3}\right)$ & $k(\%)$ & $N_{A}\left(\mathrm{~cm}^{-3}\right)$ & $E_{A}(\mathrm{eV})$ \\
\hline $\mathrm{A}$ & 6.3 & $3.5 \times 10^{16}$ & 74 & $2.5 \times 10^{16}$ & 0.57 \\
$\mathrm{~B}$ & 8.1 & $3.5 \times 10^{17}$ & 80 & $2.8 \times 10^{17}$ & 0.56 \\
$\mathrm{C}$ & 15.8 & $1.1 \times 10^{18}$ & 86 & $9.4 \times 10^{17}$ & 0.56 \\
$\mathrm{D}$ & 7.4 & $7.2 \times 10^{18}$ & 93 & $6.7 \times 10^{18}$ & 0.50 \\
\hline \hline
\end{tabular}

$3.5 \times 10^{17}$, and $1.1 \times 10^{18} \mathrm{~cm}^{-3}$. The incorporation ratio, $N_{D} /[\mathrm{P}]$, was $100 \%$ for samples A and C, but $75 \%$ for sample $\mathrm{B}$. For sample $\mathrm{D}$, as its $\mathrm{P}$ concentration is higher than the calibration limit for cathodoluminescence, we assumed $N_{D}=[\mathrm{P}]=7.2 \times 10^{18} \mathrm{~cm}^{-3}$. The sample characteristics are summarized in Table I.

Figure 2 shows the temperature dependence of the free carrier concentrations measured by the Hall effect. The data were analyzed using the general expression for the electron concentration $n$ in a partially compensated $n$-type semiconductor using Boltzmann statistics and a parabolic conduction band edge ${ }^{17}$ : $g_{d} n\left(N_{A}+n\right) /\left[N_{C}\left(N_{D}-N_{A}-n\right)\right]=\exp \left(-E_{A} / k_{B} T\right)$ with $N_{C}=2\left(2 \pi m_{d}^{*} k_{B} T / h^{2}\right)^{3 / 2}$. The degeneracy factor for the donors $g_{d}=2$. $E_{A}$ is the activation energy of the donors, $k_{B}$ is the Boltzmann constant, $h$ is the Planck constant, and $N_{C}$ is the effective density of states in the conduction band. The effective mass for the equivalent density of states $m_{d}^{*}=6^{2 / 3} m_{\|}^{1 / 3} m_{\perp}^{2 / 3}=1.639 m_{0}$, where $m_{\|}=1.56 m_{0}$ and $m_{\perp}=0.28 m_{0} \cdot{ }^{18}$ The activation energy $E_{A}$ and the compensation ratio were determined independently using the procedure described in Ref. 19 (Table I). The values of $k$ are quite large but they are state of the art for P-doped (100) oriented layers, ${ }^{8}$ for which the lowest reported $k$ is $40 \% .{ }^{10}$ The activation energy decreases for increasing $N_{D}$ or $k$, as expected from Ref. 19. The differences between the theoretical and the experimental data at the lowest temperatures are due to the hopping conduction mechanism, which is not discussed here.

Figure 3 shows the Hall electron mobility of the films as a function of temperature. The mobility curves are analyzed with the relaxation time approximation by considering electron scattering in diamond ${ }^{20,21}$ from ionized impurities (ii), intravalley acoustic phonons (ap), intervalley optical phonons (op), and neutral impurities (ni). First, we calculate the dependence of the scattering rates on the electron energy, individually for each scattering process, using the electron effective mass $m^{*}=m_{\|}^{1 / 3} m_{\perp}^{2 / 3}$.

In (100) $n$-type diamond, the compensation ratio is quite large, resulting in a large amount of ionized impurities, $N_{\mathrm{ii}}=n+2 N_{A}$. The scattering of electrons by ionized impurities is described using the Brooks-Herring model $^{22}$ (see also Chattopadhyay and Queisser ${ }^{23}$ for a review). The potential of an ionized impurity is approximated by a screened Coulomb potential. The rate of 


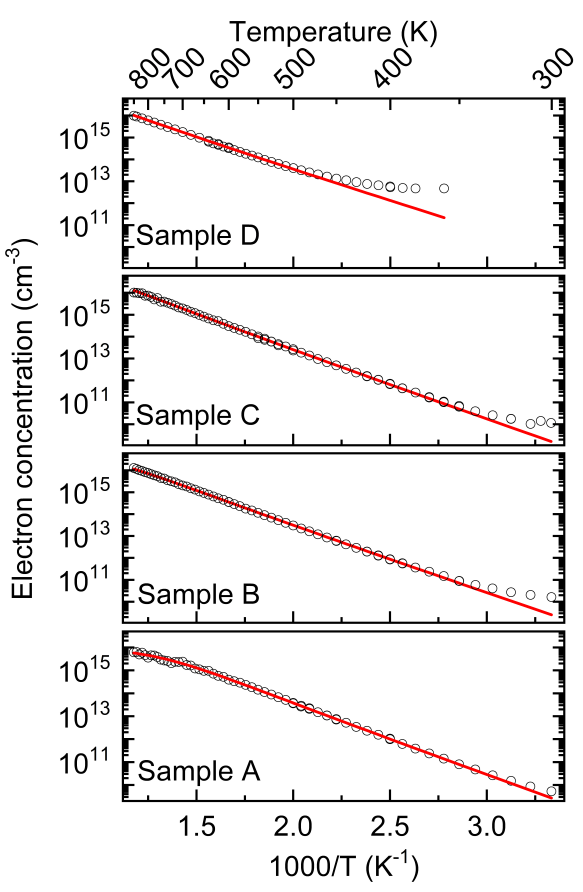

FIG. 2. Electron concentration measured by the Hall effect as a function of temperature for diamond samples A, B, C, and $\mathrm{D}$ with various $\mathrm{P}$ concentrations. Measurements have open circles, and the fit is a red line.

scattering by ionized impurities is

$$
\frac{1}{\tau_{i i}(E)}=\frac{N_{i i} q^{4}}{16 \sqrt{2} \pi \epsilon^{2} \sqrt{m^{*}}} E^{-3 / 2}\left(\ln (1+b)-\frac{b}{1+b}\right),
$$

where $b=8 m^{*} \lambda_{s}^{2} / \hbar^{2}$. The screening length for ionized impurities is a Debye screening length: $\lambda_{s}=$ $\left(\epsilon k_{B} T / q^{2} n^{\prime}\right)^{1 / 2}$ with $n^{\prime}=n+\left(N_{D}-N_{A}-n\right)\left(N_{A}+n\right) / N_{D}$ This expression takes into account the screening by ionized impurities left behind free electrons, that of electrons bound to donors, and that of ionized acceptors.

Electrons are scattered by longitudinal acoustic phonons via the deformation potential interaction. ${ }^{24}$ The process being quasi-elastic, the electrons stay in the same band. The rate of scattering by intravalley acoustic phonons is

$$
\frac{1}{\tau_{a p}}=\frac{\sqrt{2} m^{* 3 / 2} D_{a}^{2} k_{B} T}{\pi \hbar^{4} c_{1}} E^{\frac{1}{2}}
$$

where $D_{a}$ is the deformation potential constant and $c_{1}=\rho v^{2}$ is the longitudinal elastic constant. The velocity of the longitudinal phonons is $v=17536 \mathrm{~m} \mathrm{~s}^{-1}$ and the density of diamond is $\rho=3515 \mathrm{~kg} \mathrm{~m}^{-3}$. Thus, $c_{1}=1.08 \mathrm{GPa} . \quad c_{1}=c_{11}$ for a wave propagating along the (100) direction. The set of deformation potentials is an intrinsic parameter of a semiconductor, related to its

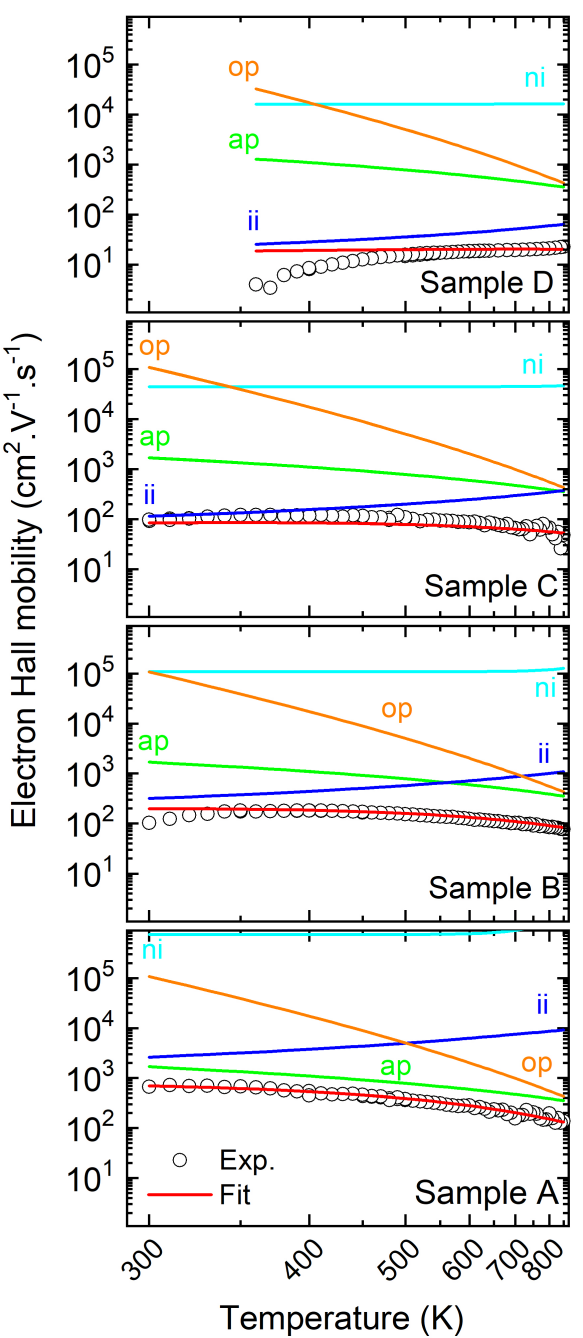

FIG. 3. Hall electron mobility in diamond as a function of temperature. Measurements have open circles. Contributions from different scattering mechanisms and the total mobility are shown as lines. Scattering is from ionized impurities (ii), acoustic phonons (ap), intervalley optical phonons (op), and neutral impurities (ni).

crystallographic directions. Here, we use an effective deformation potential associated with electron transport in the (100) plane. It is an intrinsic parameter of diamond but as diamond is anisotropic, ${ }^{25}$ its value depends on the direction of electron transport.

Electrons can also be scattered from one degenerate valley to another via intervalley scattering, which can be important due to the multivalleys of indirect bandgap semiconductors. An electron at the conduction band minimum can be scattered either into the opposite valley (g-scattering ) or into one of the four other equivalent valleys (f-scattering) by intervalley optical phonons with 
a rate:

$$
\begin{aligned}
\frac{1}{\tau_{o p}}= & \frac{4 D_{f}^{2} m^{* 3 / 2}}{\sqrt{2} \pi \rho \hbar^{3} \omega_{f}}\left[N\left(\omega_{f}\right) \sqrt{E+\hbar \omega_{f}}\right. \\
& \left.+\left(N\left(\omega_{f}\right)+1\right) \sqrt{E-\hbar \omega_{f}} U\left(E-\hbar \omega_{f}\right)\right] \\
& +\frac{4 D_{g}^{2} m^{* 3 / 2}}{\sqrt{2} \pi \rho \hbar^{3} \omega_{g}}\left[N\left(\omega_{g}\right) \sqrt{E+\hbar \omega_{g}}\right. \\
& \left.+\left(N\left(\omega_{g}\right)+1\right) \sqrt{E-\hbar \omega_{g}} U\left(E-\hbar \omega_{g}\right)\right],
\end{aligned}
$$

where $\hbar \omega_{f}=140 \mathrm{meV}$ and $\hbar \omega_{g}=165 \mathrm{meV}$ are the phonon energies, ${ }^{26}$ and $N\left(\omega_{i}\right)=1 /\left(e^{\hbar \omega_{i} / k_{B} T}-1\right)$ is the BoseEinstein distribution function. $U(x)$ is a step function, such as $U(x)=0$ for $x<0$ and $U(x)=1$ for $x \geq 0$. Furthermore, as this process is inelastic, we used the procedure of Farvacque. ${ }^{27}$ The effective deformation potentials, $D_{f}$ and $D_{g}$, are treated as adjustable parameters. Since the phonon energies are larger than $k_{B} T$ over the whole temperature range of our experiments, the sensitivity to these parameters is weak. To limit the number of adjustable parameters, we set $D_{f}=D_{g}=D_{f / g}$.

In a semiconductor like $\mathrm{Si}$ or Ge, scattering by neutral impurities plays a dominant role only at very low temperatures. The situation is different in wide bandgap semiconductors, as the concentration of neutral impurities remains significant even at temperatures above room temperature. The rate of scattering by neutral impurities is described using the model of Erginsoy ${ }^{28}$ for pentavalent impurities:

$$
\frac{1}{\tau_{n i}(E)}=\frac{20 N_{n i} \hbar a_{P}}{m^{*}},
$$

where $a_{P}$ is the Bohr radius for $\mathrm{P}$, which is estimated to be $1.4 \AA .^{29}$

The total relaxation time is calculated according to Matthiessen's rule: $\tau^{-1}(E)=\sum_{i} \tau_{i}^{-1}(E)$, where the relaxation rates are given by Eqs. (1) to (4). The Hall mobility is expressed with the usual formula:

$$
\mu_{H}=\frac{q\langle\tau\rangle}{m_{c}^{*}},
$$

where $\langle\tau\rangle=\int_{0}^{\infty} \tau E^{3 / 2} e^{-E / k_{B} T} \mathrm{~d} E / \int_{0}^{\infty} E^{3 / 2} e^{-E / k_{B} T} \mathrm{~d} E$ is the average relaxation time and $m_{c}^{*}$ is the conductivity $\operatorname{mass}\left(m_{c}^{*}=\frac{3 m_{\|} m_{\perp}}{2 m_{\|}+m_{\perp}}=0.39 m_{0}\right)$.

The electron mobilities measured for the four samples were adjusted simultaneously with Eq. (5). As shown in Fig. 3, the model correctly describes the full set of experimental data with $D_{a}=16.9 \mathrm{eV}$ and $D_{f / g}=$ $3.3 \times 10^{9} \mathrm{eV} \mathrm{cm}^{-1}$. The potentials found for the (100) orientation are close to those found by Pernot et al. for the (111) orientation $\left(D_{a}=17.7 \mathrm{eV}\right.$ and $D_{f / g}=4.2 \times$ $\left.10^{9} \mathrm{eV} \mathrm{cm}^{-1}\right) .{ }^{21}$ For both orientations, they remain large compared to those in ultra-pure diamond found using the time of flight $\left(D_{a}=12 \mathrm{eV} \text { and } D_{f / g}=4 \times 10^{8} \mathrm{eV} \mathrm{cm}^{-1}\right)^{30}$ or time-resolved cyclotron resonance $\left(D_{a}=8.7 \mathrm{eV}\right) .{ }^{31}$
There are different scattering mechanisms in the layers. The neutral impurities have a very limited effect for all samples in the whole temperature range. The maximum RT Hall mobility of this study was $\mu_{R T}=$ $670 \mathrm{~cm}^{2} \mathrm{~V}^{-1} \mathrm{~s}^{-1}$ for sample A. This value is similar to the best value reported for a sample of comparable concentration for the (111) orientation $\left(\mu_{R T}=660 \mathrm{~cm}^{2} \mathrm{~V}^{-1} \mathrm{~s}^{-1}\right.$ at $\left.N_{D}=7 \times 10^{16} \mathrm{~cm}^{-3}\right) \cdot{ }^{11}$ According to Pernot et al., due to the low compensation ratio of this sample $(k=13 \%)$, the limiting factor was only the scattering by acoustic phonons. ${ }^{21}$ In contrast, for sample A, which is (100) oriented, the scattering components for ionized impurities and acoustic phonons are of the same order of magnitude. With the higher $N_{D}$ obtained in samples B, C, and D, scattering by ionized impurities fully dominates the RT mobility.

Summarizing, the compensation by residual acceptor impurities limits the electron mobility in our (100) samples at $300 \mathrm{~K}$. Importantly, these results highlight that there is still room for improvement in the electrical properties of (100) $n$-type diamond. Indeed, reducing the compensation by residual acceptors should strongly increase the electron mobility. Many potential compensators are known, most merely from a theoretical point of view. Apart from the obvious boron acceptor, which is not present in a sufficient concentration in our samples to explain our results, they are mainly possible complexes involving hydrogen or carbon vacancies (see our previous work $\left.{ }^{13,19,32}\right)$. However, identifying the center responsible for the phosphorus compensation in diamond is experimentally difficult and still an issue. From our model, the low compensation and low doping limit of the Hall electron mobility in (100) diamond layers is $1600 \mathrm{~cm}^{2} \mathrm{~V}^{-1} \mathrm{~s}^{-1}$ at $300 \mathrm{~K}$. This value for (100) transport is closer to the electron mobilities measured by the time of flight $\left(4500 \mathrm{~cm}^{2} \mathrm{~V}^{-1} \mathrm{~s}^{-1}\right)^{33}$.

\section{CONCLUSION}

In conclusion, our systematic study of electron transport properties in a series of homoepitaxial $n$-type layers found high electron mobilities of up to $670 \mathrm{~cm}^{2} \mathrm{~V}^{-1} \mathrm{~s}^{-1}$ at room temperature, despite a rather high compensation by residual acceptor centers. The simulations of electron mobilities based on modeling the scattering mechanisms show that the electron mobility is mainly limited by ionized impurities due to compensation. These results highlight the possibility that diamond growth conditions could be optimized to achieve an electron mobility in $n$-type diamond closer to the intrinsic limit of $4500 \mathrm{~cm}^{2} \mathrm{~V}^{-1} \mathrm{~s}^{-1}$ recorded by time-of-flight measurements. 


\section{ACKNOWLEDGMENTS}

The authors would like to thank B. Berini for technical support and the French National Agency for Research for funding this work under the project MOVeToDiam No ANR-17-CE05-0019-02.

\section{DATA AVAILABILITY STATEMENT}

The data that support the findings of this study are available from the corresponding author upon reasonable request.

${ }^{1}$ S. Shikata, "Single crystal diamond wafers for high power electronics," Diamond Relat. Mater. 65, 168-175 (2016).

${ }^{2}$ S. A. Bogdanov, A. L. Vikharev, M. N. Drozdov, and D. B Radishev, "Synthesis of thick and high-quality homoepitaxial diamond with high boron doping level: Oxygen effect," Diamond Relat. Mater. 74, 59-64 (2017).

${ }^{3}$ S. Ohmagari, H. Yamada, H. Umezawa, N. Tsubouchi, A. Chayahara, and Y. Mokuno, "Growth and characterization of freestanding $\mathrm{p}+$ diamond (100) substrates prepared by hot-filament chemical vapor deposition," Diamond Relat. Mater. 81, 33-37 (2018)

${ }^{4}$ J. Achard, R. Issaoui, A. Tallaire, F. Silva, J. Barjon, F. Jomard, and A. Gicquel, "Freestanding CVD boron doped diamond single crystals: A substrate for vertical power electronic devices?" Phys. Status Solidi A 209, 1651-1658 (2012).

${ }^{5}$ V. Mortet, M. Daenen, T. Teraji, A. Lazea, V. Vorlicek, J. D'Haen, K. Haenen, and M. D'Olieslaeger, "Characterization of boron doped diamond epilayers grown in a NIRIM type reactor," Diamond Relat. Mater. 17, 1330-1334 (2008).

${ }^{6}$ S. Ri, H. Kato, M. Ogura, H. Watanabe, T. Makino, S. Yamasaki, and H. Okushi, "Electrical and optical characterization of boron-doped (111) homoepitaxial diamond films," Diamond Relat. Mater. 14, 1964-1968 (2005).

${ }^{7}$ H. Kato, M. Ogura, T. Makino, D. Takeuchi, and S. Yamasaki, " $n$-type control of single-crystal diamond films by ultralightly phosphorus doping," Appl. Phys. Lett. 109 (2016), 10.1063/1.4964382.

${ }^{8}$ H. Kato, T. Makino, S. Yamasaki, and H. Okushi, " $n$-type diamond growth by phosphorus doping," in Diamond Electronics Fundamentals to Applications II (Cambridge University Press, 2008), pp. 39-48.

${ }^{9}$ H. Kato, J. Barjon, N. Habka, T. Matsumoto, D. Takeuchi, H. Okushi, and S. Yamasaki, "Energy level of compensator states in (001) phosphorus-doped diamond," Diamond Relat. Mater. 20, 1016-1019 (2011).

${ }^{10}$ M.-A. Pinault-Thaury, I. Stenger, F. Jomard, J. Chevallier, J. Barjon, A. Traore, D. Eon, and J. Pernot, "Electrical activity of (100) $n$-type diamond with full donor site incorporation of phosphorus," Phys. Status Solidi A 212, 2454-2459 (2015).

${ }^{11}$ M. Katagiri, J. Isoya, S. Koizumi, and H. Kanda, "Lightly phosphorus-doped homoepitaxial diamond films grown by chemical vapor deposition," Appl. Phys. Lett. 85, 6365-6367 (2004).

${ }^{12}$ T. Kociniewski, M.-A. Pinault, J. Barjon, F. Jomard, J. Chevallier, and C. Saguy, "MOCVD doping technology for phosphorus incorporation in diamond: Influence of the growth temperature on the electrical properties," Diamond Relat. Mater. 16, 815-818 (2007).

${ }^{13}$ M. A. Pinault-Thaury, B. Berini, I. Stenger, E. Chikoidze, A. Lusson, F. Jomard, J. Chevallier, and J. Barjon, "High fraction of substitutional phosphorus in a (100) diamond epilayer with low surface roughness," Appl. Phys. Lett. 100 (2012), $10.1063 / 1.4712617$
${ }^{14}$ N. Temahuki, R. Gillet, V. Sallet, F. Jomard, E. Chikoidze, Y. Dumont, M.-A. Pinault-Thaury, and J. Barjon, "New process for electrical contacts on (100) n-type diamond," Phys. Status Solidi A 214 (2017), 10.1002/pssa.201700466.

${ }^{15} \mathrm{~J}$. Barjon, P. Desfonds, M.-A. Pinault, T. Kociniewski, F. Jomard, and J. Chevallier, "Determination of the phosphorus content in diamond using cathodoluminescence spectroscopy," J. Appl. Phys. 101 (2007), 10.1063/1.2735408.

${ }^{16}$ J. Barjon, M.-A. Pinault, T. Kociniewski, F. Jomard, and J. Chevallier, "Cathodoluminescence as a tool to determine the phosphorus concentration in diamond," Phys. Status Solidi A 204, 2965-2970 (2007).

${ }^{17}$ J. S. Blakemore, Semiconductor Statistics, edited by H. K. Henisch (Pergamon Press, 1962).

${ }^{18}$ N. Naka, K. Fukai, Y. Handa, and I. Akimoto, "Direct measurement via cyclotron resonance of the carrier effective masses in pristine diamond," Phys. Rev. B 88 (2013), 10.1103/PhysRevB.88.035205.

${ }^{19}$ I. Stenger, M. A. Pinault-Thaury, T. Kociniewski, A. Lusson, E. Chikoidze, F. Jomard, Y. Dumont, J. Chevallier, and J. Barjon, "Impurity-to-band activation energy in phosphorus doped diamond," J. Appl. Phys. 114 (2013), 10.1063/1.4818946.

${ }^{20}$ J. Pernot, W. Zawadzki, S. Contreras, J. Robert, E. Neyret, and L. Di Cioccio, "Electrical transport in $n$-type $4 \mathrm{H}$ silicon carbide," J. Appl. Phys. 90, 1869-1878 (2001).

${ }^{21}$ J. Pernot, C. Tavares, E. Gheeraert, E. Bustarret, M. Katagiri, and S. Koizumi, "Hall electron mobility in diamond," Appl. Phys. Lett. 89 (2006), 10.1063/1.2355454.

${ }^{22}$ H. Brooks, "Scattering by ionized impurities in semiconductors," Phys. Rev. 83, 879 (1951)

${ }^{23}$ D. Chattopadhyay and H. J. Queisser, "Electron-scattering by ionized impurities in semiconductors," Rev. Mod. Phys. 53, 745768 (1981).

${ }^{24} \mathrm{P}$. Yu and M. Cardona, Fundamentals of Semiconductors: Physics and Materials Properties, 4th ed., Graduate Texts in Physics (Springer, 2010) pp. 1-775.

${ }^{25} \mathrm{C}$. Herring and E. Vogt, "Transport and deformation-potential theory for many-valley semiconductors with anisotropic scattering," Phys. Rev. 101, 944-961 (1956).

${ }^{26}$ S. A. Solin and A. K. Ramdas, "Raman spectrum of diamond," Phys. Rev. B 1, 1687-\& (1970).

${ }^{27} \mathrm{~J}$. Farvacque, "Extension of the collision-time tensor to the case of inelastic scattering mechanisms: Application to GaAs and GaN," Phys. Rev. B 62, 2536-2541 (2000).

${ }^{28}$ C. Erginsoy, "Neutral impurity scattering in semiconductors," Phys. Rev. 79, 1013-1014 (1950).

${ }^{29}$ B. Butorac and A. Mainwood, "Symmetry of the phosphorus donor in diamond from first principles," Phys. Rev. B 78 (2008), 10.1103/PhysRevB.78.235204.

${ }^{30}$ J. Hammersberg, S. Majdi, K. K. Kovi, N. Suntornwipat, M. Gabrysch, D. J. Twitchen, and J. Isberg, "Stability of polarized states for diamond valleytronics," Appl. Phys. Lett. 104 (2014), 10.1063/1.4882649.

${ }^{31}$ I. Akimoto, Y. Handa, K. Fukai, and N. Naka, "High carrier mobility in ultrapure diamond measured by time-resolved cyclotron resonance," Appl. Phys. Lett. 105 (2014), 10.1063/1.4891039.

${ }^{32}$ I. Stenger, M.-A. Pinault-Thaury, A. Lusson, T. Kociniewski, F. Jomard, J. Chevallier, and J. Barjon, "Quantitative analysis of electronic absorption of phosphorus donors in diamond," Diamond Relat. Mater. 74, 24-30 (2017).

${ }^{33} \mathrm{~J}$. Isberg, J. Hammersberg, E. Johansson, T. Wikstrom D. Twitchen, A. Whitehead, S. Coe, and G. Scarsbrook, "High carrier mobility in single-crystal plasma-deposited diamond," Science 297, 1670-1672 (2002).

${ }^{34}$ J. Barjon, E. Chikoidze, F. Jomard,Y. Dumont, M.-A. PinaultThaury, R. Issaoui, O. Brinza, J. Achard and F. Silva, "Homoepitaxial boron-doped diamond with very low compensation," Phys. Status Solidi A 9, 1750-1753 (2012). 
(a)

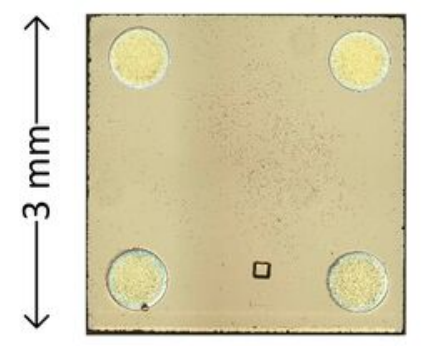

(b)

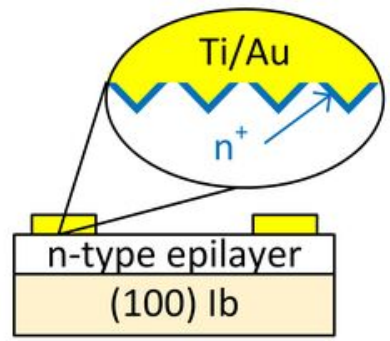

(c)

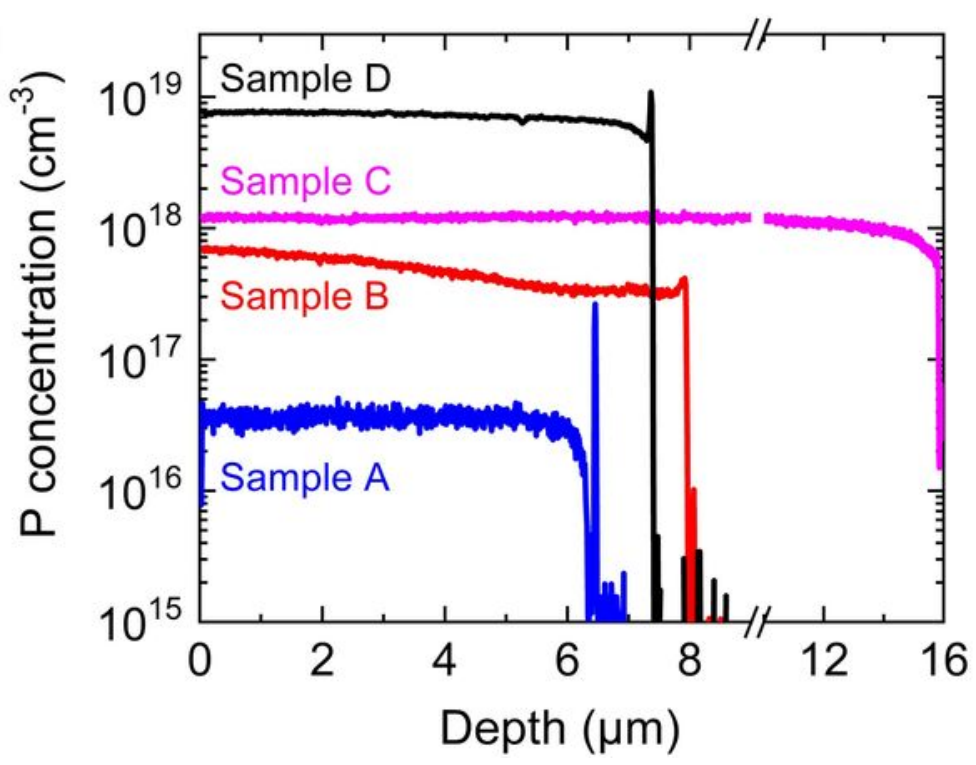




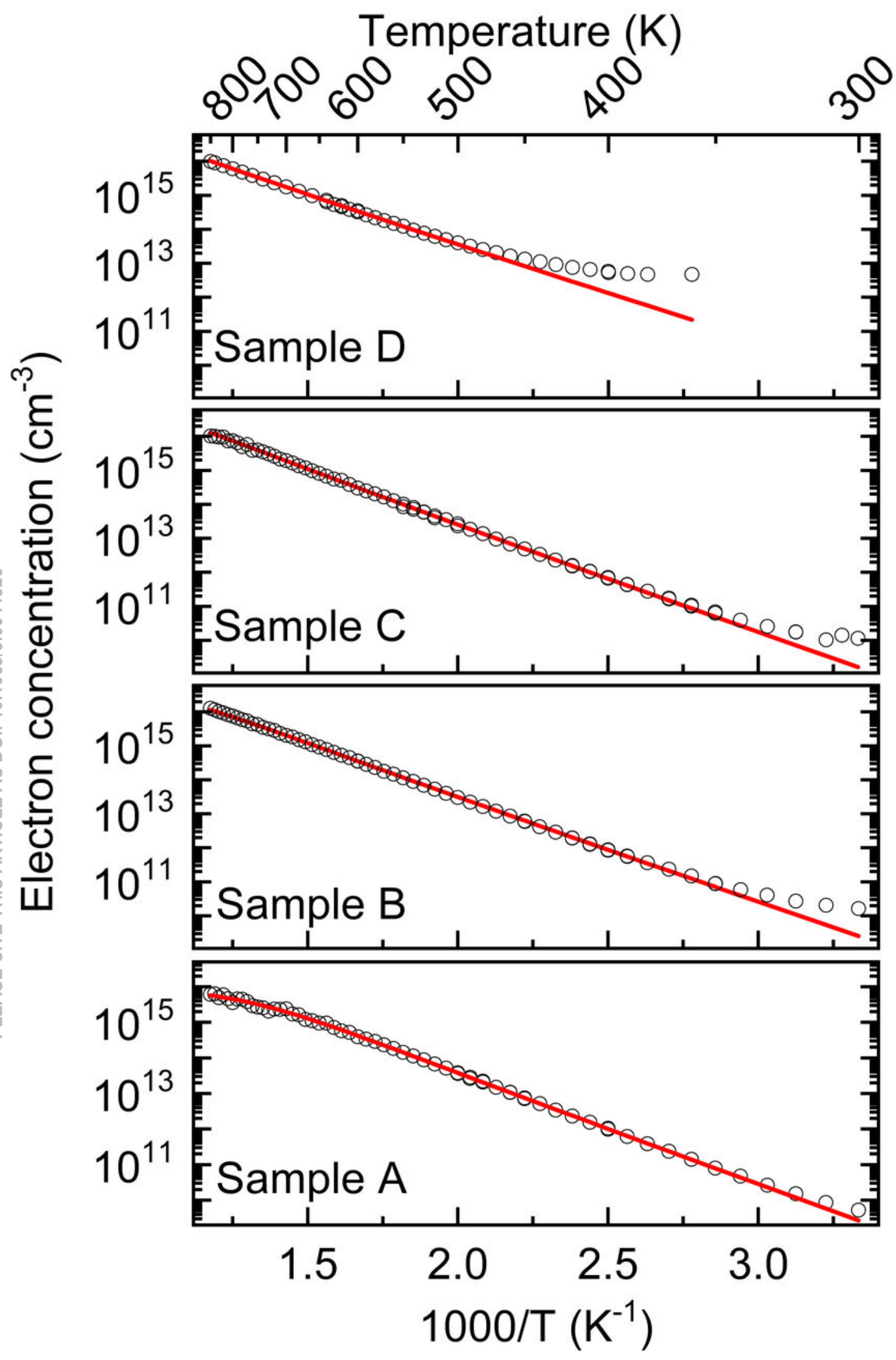


